\title{
El comportamiento innovador en empresas: una visión desde la autonomía y la disponibilidad de tiempo de los empleados de una empresa del sector transporte en Tamaulipas \\ Innovative behavior in companies: a vision from the autonomy and time availability of employees of a company in the transport sector in Tamaulipas
}

Esthela Galván Vela ${ }^{1}$, Yesenia Sánchez Tovar ${ }^{2}$ y Mónica Lorena Sánchez Limón ${ }^{2}$

Palabras clave: innovación; autonomía; disponibilidad de tiempo; empresas de servicios Keywords: innovation; autonomy; time availability; service companies

Recibido en: 19-10-2019 / Aceptado en: 21-01-2020

\section{Resumen}

Introducción: Las condiciones dinámicas de los mercados demandan el estudio de la innovación empresarial. Con base en el estudio de la literatura de gestión, se ha propuesto que existe una influencia de los factores organizacionales de autonomía y disponibilidad de tiempo otorgado a los empleados en el nivel de innovación en la empresa.

Método: Para probar esta suposición, se aplicó un instrumento cuantitativo elaborado a partir de la escala CEAI y la medida ENTRESCALE, considerando 144 observaciones en una empresa de servicios de transporte de pasajeros en el norte de México. Posteriormente, se realizó un análisis factorial exploratorio para verificar la validez de los constructos: el alfa de Cronbach para identificar su nivel de confiabilidad; y la técnica de regresión lineal múltiple, a fin de verificar el efecto de las variables independientes sobre la innovación.

Resultados: El análisis factorial exploratorio y la medida del alfa de Cronbach respaldan la validez de constructo y la confiabilidad de los ítems que componen el instrumento de medida. Los resultados de la técnica de regresión lineal múltiple demuestran que solo la autonomía tiene un efecto con el nivel de innovación en el sector, por lo que se descarta que la disponibilidad de tiempo libre se relacione con la conducta innovadora.

Discusión o Conclusión: Los resultados contribuyen al desarrollo teórico de los constructos, e invitan a la reflexión sobre mejores prácticas empresariales que deriven en el desarrollo de una conducta innovadora en la empresa.

\footnotetext{
${ }^{1}$ Centro de Enseñanza Técnica y Superior, Escuela de Administración y Negocios. Tijuana, Baja California, México. E-mail: gavela2012@gmail.com

${ }^{2}$ Universidad Autónoma de Tamaulipas, Facultad de Comercio y Administración, Victoria, Tamaulipas, México

(C) Universidad De La Salle Bajío (México)
} 
El comportamiento innovador en empresas: una visión desde la autonomía y la disponibilidad de tiempo de los empleados de una empresa del sector transporte en Tamaulipas

\begin{abstract}
Introduction: The dynamic conditions of the markets demand the study of business innovation. Based on the study of management literature, it has been proposed that there is a relationship between the organizational factors of autonomy and time availability granted to employees with the level of innovation in the company.

Method: To prove this assumption a quantitative instrument was developed based on the CEAI scale and the ENTRESCALE measure and 144 observations were collected from a passenger transport services company in northern Mexico. Subsequently, an exploratory factor analysis was performed to verify the validity of the constructs; Cronbach's alpha to identify its level of reliability and the multiple linear regression technique in order to verify the effect of independent variables on innovation.
\end{abstract}

Results: The exploratory factor analysis and Cronbach's alpha measurement support the construct validity and reliability of the items that make up the measuring instrument. The results of the multiple linear regression technique show that only autonomy is related to the level of innovation in the sector, so it is ruled out that the availability of free time is related to innovative behavior.

Discussion or Conclusion: The results contribute to the theoretical development of the constructs and invite reflection on best business practices that lead to the development of innovative behavior in the company.

\title{
Introducción
}

Durante los últimos años se ha incrementado la búsqueda de formas alternativas de abordar los problemas de crecimiento y competitividad que demandan las empresas inmersas en la dinámica comercial de las economías emergentes (Galván-Vela y Sánchez-Limón, 2017, 2019). En este sentido, la línea de discusión se ha centrado en el papel que juega la innovación sobre la productividad, el crecimiento y el desarrollo social y económico de los entes que le producen (Halme, Lindeman y Linna, 2012; Turro, Urbano y Peris-Ortíz, 2014).

Según Laclette (2012), los países que revelan un mayor desempeño en sus índices de crecimiento y desarrollo económico, son aquellos que realizan las inversiones más significativas en materia de ciencia, tecnología e innovación. Se presume que este efecto no es meramente una condición macroeconómica sino también puede ser aplicable al sector empresarial en donde se ha demostrado la influencia positiva de la innovación en la productividad, la competitividad, el 
crecimiento relativo o absoluto, la revitalización y el desarrollo financiero de la empresa (Baruah y Ward, 2014; Parker, 2011; Turró et al., 2014).

Particularmente en lo que se refiere a la innovación empresarial existen vertientes teóricas relacionadas con el estudio de la influencia de las condiciones exógenas, los elementos endógenos y de las consecuencias de la innovación en la empresa (Martin, 2016). Por esto, un área de oportunidad importante en el estudio de la innovación es aquella relacionada con el efecto de los actos intrafirma, como la postura estratégica de la empresa, el desarrollo de nuevos modelos de negocio o el papel de las personas involucradas en el proceso (Anderson y Markides, 2007; Halme et al., 2012).

Por lo anterior resulta imperativo el conocimiento del efecto de los factores internos relacionados a la innovación a fin de subrogar las capacidades incipientes de la empresa actual y contribuir en el desarrollo de mejores prácticas empresariales (Kuratko, Hornsby y Covin, 2014; Parker, 2011), adicionalmente, el estudiar la innovación a partir de sus predictores permite la generación de conocimiento en un área de interés en la que aún se requieren aportaciones teóricas (Hornsby, Kuratko, Shepherd y Bott, 2009).

En consecuencia, se propone el estudio del comportamiento innovador a partir de dos condiciones relacionadas con la postura estratégica de la empresa y el estilo gerencial de la misma: la autonomía brindada al empleado y el tiempo libre (disponibilidad de tiempo) que se les otorga para el desarrollo creativo. Por lo que los objetivos de la presente investigación son: 1) identificar la relación existente entre el nivel de autonomía otorgada a los empleados con la innovación empresarial; 2) identificar la relación existente entre el tiempo libre brindado a los empleados para el desarrollo creativo con la innovación empresarial. Ambos objetivos se lograron en una empresa de servicios de transporte de pasajeros cuyas operaciones se desarrollan en el noreste de México y cuya sede central se encuentra en la capital del estado de Tamaulipas.

La razón principal para el análisis de estas presuntas condicionantes de la innovación en una empresa de servicios de transporte de pasajeros es contribuir a la generación de teoría en un sector que pese a su potencial competitivo, ha sido relativamente olvidado en el sentido de que la investigación en la materia se aboca al estudio de las competencias o dinámicas empresariales relacionadas a la innovación en sectores industriales como el automotriz, el químico o el de manufactura de sistemas de cómputo; situación que se hace presente también en materia de datos descriptivos en el que se manifiestan evidentes deficiencias (Michelli \& Oliver, 2017). 
En el marco de estos planteamientos es relevante enunciar también que la literatura especializada en la materia propone el estudio de los factores asociados a la innovación desde la referencia de situaciones concretas en territorios y sectores específicos, esto a fin de seguir la lógica que demanda el estudio del fenómeno en empresas y mercados heterogéneos (e.g. Antoncic, 2007; Kuratko et al., 2014).

\section{Comportamiento innovador}

Uno de los principales referentes en materia de innovación y emprendimiento es el economista austriaco Joseph Schumpeter (Antoncic, 2007; Guth y Ginsberg, 1990; Miller, 1983; Turró et al., 2014; Zahra, 1991, 1993) quien a grandes rasgos consideró que la producción es el resultado de una combinación de fuerzas materiales e inmateriales. Las primeras están compuestas por los factores de producción comunes (tierra, trabajo y capital); y las segundas engloban elementos de la organización (tecnología e innovación y aspectos socioculturales). Estos últimos, fueron llamados también factores de la evolución económica y representan un impacto más dinámico y decisivo en las empresas.

Si bien por aquel entonces no se reconocían ambas fuerzas como recursos y capacidades empresariales, para Schumpeter, estos también determinaban tanto la naturaleza de la organización como sus resultados económicos. En este sentido el cambio organizacional podía ser descrito como un proceso endógeno derivado de las condiciones exógenas. La Teoría del Desenvolvimiento Económico (TDE) de Schumpeter (1934) por ejemplo, ejemplificaba el cambio organizacional por medio de la dinámica del desarrollo y las combinaciones de aprendizaje existente e incremental en la empresa.

Los cambios radicales derivados de tal interacción fueron descritos como innovaciones, incluyendo entre los mismos: la introducción de nuevos productos y servicios; la apertura y explotación de nuevos mercados; el desarrollo de nuevos métodos de producción o logística; los cambios revolucionarios en la estructura de la organización, en sus métodos de gestión o en sus procesos y la generación de nuevas fuentes de materia prima como las alianzas, la reubicación y la creación de redes de colaboración.

Según las premisas fundamentales de la TDE estas innovaciones pueden ser consideradas parte de la "destrucción creativa", entendida como el proceso en el cual los individuos con características emprendedoras son capaces de introducir nuevas combinaciones de recursos 
empresariales bajo condiciones de desequilibrio en el mercado -derivados de su estructura y grado de competitividad-. Es en este quiebre de las condiciones de equilibrio en los diferentes sectores en que se manifiesta la participación activa del empresario innovador, es decir, aquél individuo que propicia la innovación en la empresa por medio de la combinación del conocimiento actual e incremental.

En este sentido, el desarrollo económico no está dado por la espontaneidad o por el mero resultado de las condiciones exógenas, sino por la base de conocimientos previos en la empresa y la promoción activa de estos empresarios innovadores que, dotados de capacidades para el oportuno aprovechamiento de los recursos de la empresa, son dados a innovar y a competir en condiciones de incertidumbre ambiental. Lo anterior se manifiesta en el reconocimiento de la existencia de combinaciones innovadoras de capacidades que poseen algunas empresas y que otras, no pueden igualar (Schumpeter, 1942).

Las aportaciones anteriormente descritas fueron relegadas en la literatura de gestión durante las décadas posteriores a su desarrollo (Yoguel, Barletta y Pereira, 2013). No obstante, gracias a autores como Nelson y Winter $(1982,2002)$ y Teece y Pisano (1994) y colaboradores (1997), es que surge un pensamiento neoshumpeteriano en torno a la innovación y las capacidades dinámicas. Por ende, en las últimas décadas se han desarrollado estudios relacionados al esclarecimiento de factores asociados con la naturaleza, la conducta y el desempeño de la organización desde la perspectiva de sus capacidades internas, más allá de la noción propiamente clásica que pretendía el estudio de la empresa por medio de la evaluación del comportamiento de una industria compuesta por entidades homogéneas (Hoskisson, Hitt, Wan y Yiu, 1999; Nelson, 1991; Nelson y Winter, 2002).

En tanto lo anterior, la Organización para la Cooperación y el Desarrollo Económico (OCDE) y la Oficina de Estadística para las Comunidades Europeas (EUROSTAT), en respuesta al aumento en el desarrollo de modelos y marcos analíticos para la innovación desarrollados a nivel global, elaboraron por primera vez en 1992 el Manual de Oslo, el cual ha simbolizado hasta ahora el referente más significativo en materia de innovación empresarial, sobre todo en cuanto a la conceptualización, tipología y trazado de indicadores en el rubro (OCDE, 2005).

La terminología con relación a tal dimensión es vasta. No obstante, la OCDE y el EUROSTAT (2005) propusieron por medio del Manual de Oslo una definición amplia al término “innovación”, que involucra los cambios previstos en las actividades de las organizaciones 
El comportamiento innovador en empresas: una visión desde la autonomía y la disponibilidad de tiempo de los empleados de una empresa del sector transporte en Tamaulipas

orientados a la mejora de sus resultados, resaltando en el mismo algunas características necesarias para estos cambios. Estas son:

1) La asociación de la innovación con la incertidumbre.

2) La necesidad de recursos para la inversión en innovación; adquisición de activos materiales o inmateriales.

3) La posibilidad de la relación entre la innovación y el Spillover; desbordamiento tecnológico.

4) La implicación del uso de un nuevo conocimiento o la combinación de conocimientos existentes.

5) La innovación está orientada a resultados como la obtención de ventajas competitivas, el desplazamiento positivo de la curva de demanda de un bien o el desplazamiento negativo de los costos.

6) La existencia de actividades específicas de la innovación, como la investigación y desarrollo (I+D), gestión de derechos y patentes, gestión del conocimiento o reingeniería.

En relación con lo anterior y con base en evidencia empírica en torno al enunciado constructo, el presente estudio entiende como un comportamiento innovador a la capacidad de la empresa para el desarrollo de actos de diseño y soporte a las ideas relacionados a nuevos productos y servicios a partir de prácticas que involucren procesos creativos y tecnología (Antonic y Hisrich, 2001; Antonic, 2007; Kearney, Hisrich y Antoncic, 2013).

\section{Autonomía y disponibilidad de tiempo}

Múltiples aportaciones teórico-científicas señalan la importancia de elementos internos que inciden de forma directa en el comportamiento innovador (e.g. Alpkan Bulut, Gunday, Ulusoy y Kilic, 2010; Antoncic, 2007; Ireland, Covin y Kuratko, 2009; Kearney, Hisrich y Roche, 2010; Kearney, Hisrich y Antoncic, 2013; Kuratko y Audretsch, 2013; Kuratko et al., 2014). Estos elementos involucran el cúmulo de actos o conductas surgidas en el interior de la empresa entre los que destacan aspectos culturales y filosóficos, de estructura y de estrategia o de estilos gerenciales y métodos de toma de decisión (Antoncic y Hisrich, 2001; De Villiers-Scheepers, 2012). Sin embargo, el primer acercamiento al estudio de las variables independientes de esta 
investigación ha sido el modelo de Hornsby, Kuratko y Montagno (1999), quienes propusieron a la autonomía y la disponibilidad de tiempo como elementos relacionados al emprendimiento corporativo caracterizado por la innovación empresarial, como una continuación a la propuesta inicial de Kuratko, Montagno y Hornsby (1990) quienes operacionalizaron los factores organizacionales en soporte de la administración, estructura de apoyo y las recompensas.

En lo que respecta específicamente a la autonomía, Kuratko, Ireland, Covin y Hornsby (2005) se refieren a la misma como el “...compromiso de los gerentes de alto nivel de tolerar el fracaso, proporcionar libertad de decisión y libertad de supervisión excesiva [más allá de lo que se considera razonable] y delegar autoridad y responsabilidad a los gerentes de nivel intermedio" (p. 703), por tanto, el proporcionar ciertos niveles de autonomía permite la descentralización del poder en la toma de decisiones.

Para Kuratko (2010) es imperativo considerar que la postura de los mandos altos con relación a la empresa no afecte la libertad o delegación de responsabilidades a los mandos inferiores, quienes convenientemente no deberían estar sujetos a supervisiones constantes. Así mismo, Alpkan et al. (2010) señalaron que la autonomía se refleja en el grado de motivación brindado a los empleados para tomar decisiones con respecto a su trabajo y la conceptualizan como “...el grado de iniciativa de los empleados en su trabajo formal y la implementación de esfuerzos de mejora o resolución de problemas" (p. 736).

La autonomía como constructo se ha abordado desde décadas atrás en la literatura de gestión. Por ejemplo Morse (1986), enunció que un ambiente organizacional que sea percibido con ciertos niveles de autonomía es capaz de retener y explotar el talento innovador del personal, pues una fuerte motivación emprendedora es el hecho de brindar a los empleados cierto grado de libertad.

Investigaciones empíricas han tratado de evidenciar la relación existente entre el mencionado constructo con cuestiones referentes a la innovación y el emprendimiento en la empresa. Por ejemplo, Hornsby et al. (2009) encontraron que el proporcionar autonomía en las decisiones y acciones de los empleados se encuentra asociado de forma positiva y significativa con la cantidad de nuevas ideas desarrolladas en pro de la organización. Así mismo, Wyk y Adonisi (2012) encontraron una relación positiva y significativa entre la autonomía y el nivel de emprendimiento en la empresa. 
Por su parte, la disponibilidad de tiempo es otro de los factores organizacionales propuestos por Hornsby et al. (1999) como uno de los principales detonantes en el desarrollo del comportamiento innovador en la empresa. Su estudio data desde los primeros aportes teóricos de Burgelman (1984), quien reconoció al tiempo libre como un constructo que permitía el desarrollo de innovaciones en la empresa.

Más adelante, Duncan, Ginter, Rucks y Jacobs (1988) argumentaron que las empresas con altos controles formales o muy burocratizadas tienden a limitar el comportamiento creativo e innovador de la plantilla de trabajo. Contrario a estas limitaciones, empresas como 3M han lanzado exitosamente al mercado productos por iniciativa de su plantilla laboral como resultado de brindar el tiempo necesario a los empleados para realizar sus actividades y un porcentaje de tiempo adicional para el desarrollo de ideas propias.

En tanto lo anterior, se tiene que la disponibilidad de tiempo es una variable que se relaciona con la carga de horarios que los empleados poseen para el desarrollo de sus actividades diarias y el tiempo que les queda disponible dentro de su jornada para la procuración de las nuevas ideas (Kuratko et al., 2014). Este constructo sugiere a su vez una evaluación de personal o individual sobre la posibilidad de contar con lapsos suficientes para el desarrollo de innovaciones (Kuratko et al., 2005). En este sentido, el aprovechamiento oportuno de las condiciones munificentes del entorno está en función del tiempo libre que los empleados posean, pues sólo de esta forma estarían propensos a captar las oportunidades que de otra forma no habrían podido percibir (Kuratko et al., 2014). Lo anterior se fundamenta en diversas investigaciones como la de Hornsby, Kuratko, Holt y Wales (2013), quienes encontraron correlaciones positivas y significativas entre la disponibilidad de tiempo en los empleados y el desarrollo de innovaciones en la empresa.

\section{Método}

La revisión teórica de los constructos a los que se hace alusión en el primer apartado de esta investigación originó la propuesta de dos hipótesis: H1) Proporcionar autonomía a los empleados afecta de forma positiva y significativa el nivel de innovación en la empresa y H2) Proporcionar tiempo libre a los empleados afecta de forma positiva y significativa el nivel de innovación en la empresa. 
Cabe señalar que la presente investigación atiende al paradigma cuantitativo en cuanto a su recolección, procesamiento y análisis de datos. El diseño de la misma es no experimental y transversal, puesto que las variables no fueron sometidas a manipulación alguna y las observaciones fueron recolectadas en un único momento en el tiempo (Kerlinger y Lee, 1983).

Las observaciones de esta investigación fueron recolectadas en una empresa grande que otorga el servicio de transporte de pasajeros por vía terrestre en toda la zona noreste de México y su sede se encuentra en ciudad Victoria Tamaulipas. Se consideraron para el estudio a la totalidad de los empleados de mandos medios y se descartaron a quienes laboran en áreas de calidad y operativas, según las recomendaciones de De Villiers-Scheepers (2012), en el sentido de que estas áreas presentan labores estandarizadas y poco proclives al desarrollo de innovaciones.

Tras una depuración de cuestionarios se analizaron 144 observaciones, las cuales corresponden al 57\% de los trabajadores de la empresa en cuestión. El instrumento utilizado para la medición del nivel de autonomía y la disponibilidad de tiempo consistió en una transcripción y adecuación del Instrumento de Evaluación de Emprendimiento Corporativo (CEAI), el cual fue propuesto originalmente por Hornsby, Kuratko y Zahra (2002) y perfeccionado y validado en investigaciones posteriores por Kuratko et al. (2014). Para medir la innovación, se tomaron como referencia los ítems que miden el grado de orientación innovadora en la ENTRESCALE de Knight (1997). En total, la medición de los tres constructos del estudio se realizó por un cuestionario compuesto de 20 ítems cuya distribución fue: para autonomía, 10 ítems; para disponibilidad de tiempo, 6 ítems; y para innovación 4 ítems.

El procedimiento de análisis de datos consistió en una serie de pruebas a fin de verificar la confiabilidad y validez de los constructos de la escala, entre ellas, el análisis factorial exploratorio (AFE) por el método de componentes principales con rotación Varimax y el Alpha de Cronbach. Un segundo ejercicio de análisis de correlación permitió identificar correlaciones significativas en las variables en tanto que $\mathrm{p}<0.05 \mathrm{y}$, por último, un tercer ejercicio consistió en el análisis de regresión lineal múltiple a fin de verificar el efecto de las variables independientes sobre la innovación.

\section{Resultados}

Con la finalidad de comprobar las hipótesis anteriormente planteadas se realizó como primer paso un análisis factorial exploratorio para las variables independientes del estudio por medio del 
método de componentes principales con rotación Varimax. En el mencionado análisis el test de esfericidad de Barlett mostró una significatividad de 0.000, por lo que se asume previamente la correlación entre los ítems que componen los constructos, así como la aplicabilidad del análisis factorial para la agrupación de las variables en tanto $p<0.05$. Así mismo, la prueba Kaiser, Meyer y Olkin (KMO) indicó como una primera aproximación la existencia de correlaciones significativas entre los constructos y una adecuación muestral conveniente al señalar un valor por encima del mínimo aceptable (0.500), catalogado como "sobresaliente" en un 0.764 (Hair, Anderson, Thatam y Black, 1999; Pérez, 2009).

Cuadro 1. Pruebas KMO y esfericidad de Barlett. Chart 1. KMO test and Barlett's sphericity.

\begin{tabular}{|lll|}
\hline Medida Kaiser-Meyer-Olkin & 0.764 \\
Prueba de & Aprox. Chi-cuadrado & 448.488 \\
esfericidad de & Gl & 36 \\
Bartlett & Sig. & 0.000 \\
\hline
\end{tabular}

Fuente: Elaboración propia, a partir de datos arrojados en SPSS V. 24. Source: Own elaboration, based on data provided in SPSS V. 24.

En cuanto a la agrupación por medio de componentes principales (cuadro 2), para las variables independientes de este estudio, se han encontrado dos factores que marcadamente representan la autonomía y la disponibilidad de tiempo. No obstante, de los 16 ítems seleccionados para su medición por medio del CEAI, se descartaron siete ítems, es decir: F1.2, F1.3, F1.4 y F1.5 de la variable "autonomía" y F2.2, F2.3 y F2.6 de la variable disponibilidad de tiempo. Las razones por las que fueron descartados dichos ítems fueron: 1) presentaban bajas comunalidades o 2) no contribuían a la medición de su respectivo constructo al presentar cargas factoriales menores a 0.500 .

El cuadro 2 muestra, además de los factores agrupados, el porcentaje explicado de la varianza de los dos constructos en donde la autonomía explica un 39.15\% y la disponibilidad de tiempo un 19.77\% con respecto al fenómeno de innovación en la empresa. Así mismo, se muestran datos válidos a nivel de constructo al presentarse valores en alfa de Cronbach en 0.842 y 0.654, respectivamente, lo cual, según la propuesta de George y Mallery (2003) indica un grado de confiabilidad "buena" para autonomía y "cuestionable" con relación a la disponibilidad de tiempo. 
Cuadro 2. Matriz de componentes rotados.

Chart 2. Rotated component matrix.

\begin{tabular}{|c|c|c|c|}
\hline Variable & Ítems & Factor 1 & Factor 2 \\
\hline \multirow{6}{*}{ Autonomía } & F1.1. Siento que soy mi propio jefe y no tengo que revisar todas mis decisiones. & 0.700 & 0.019 \\
\hline & F1.6. Tengo la libertad de decidir lo que hago en mi trabajo. & 0.778 & 0.007 \\
\hline & $\begin{array}{l}\text { F1.7. Es básicamente mi propia responsabilidad el decidir cómo se hace mi } \\
\text { trabajo. }\end{array}$ & 0.760 & 0.124 \\
\hline & F1.8. Casi siempre decido qué hago en mi trabajo. & 0.838 & 0.207 \\
\hline & $\begin{array}{l}\text { F1.9. Tengo mucha autonomía en mi trabajo y me quedo sólo para hacer mi } \\
\text { propio trabajo. }\end{array}$ & 0.699 & 0.340 \\
\hline & $\begin{array}{l}\text { F1.10. Rara vez tengo que seguir los mismos métodos de trabajo o pasos para } \\
\text { hacer mis tareas principales de un día a otro. }\end{array}$ & 0.671 & -0.246 \\
\hline \multirow{3}{*}{$\begin{array}{l}\text { Disponibilidad } \\
\text { de Tiempo }\end{array}$} & $\begin{array}{l}\text { F2.1. Durante los últimos } 3 \text { meses, mi carga de trabajo fue demasiado pesada para } \\
\text { dedicar tiempo a desarrollar nuevas ideas. }\end{array}$ & -0.071 & 0.738 \\
\hline & $\begin{array}{l}\text { F2.4. Mi trabajo está estructurado de manera que tengo muy poco tiempo para } \\
\text { pensar en problemas mayores de la organización. }\end{array}$ & 0.208 & 0.741 \\
\hline & $\begin{array}{l}\text { F2.5. Siento que siempre estoy trabajando con limitaciones de tiempo en mi } \\
\text { trabajo. }\end{array}$ & 0.053 & 0.782 \\
\hline \multirow{3}{*}{$\begin{array}{c}\text { Otros } \\
\text { indicadores }\end{array}$} & Auto-valores & 3.524 & 1.78 \\
\hline & Porcentaje de la varianza & 39.154 & 19.772 \\
\hline & Alpha de Cronbach & 0.842 & 0.654 \\
\hline
\end{tabular}

Fuente: Elaboración propia a partir de datos arrojados en SPSS V. 24.

Source: Own elaboration based on data provided in SPSS V. 24.

En cuanto a las pruebas para la variable de comportamiento innovador, se tiene que el test de esfericidad de Barlett muestra una significatividad en 0.000 , por lo que también se asume la aplicabilidad del análisis de fiabilidad para verificar la agrupación de los ítems que pretenden medir esta variable en un componente. Así mismo, la medida KMO indica un valor catalogado como "sobresaliente" en la propuesta de Hair et al. (1999).

Cuadro 3. Pruebas KMO y Barlett para variable de innovación.

Chart 3. KMO test and Barlett's sphericity for innovation variable.

\begin{tabular}{|llc|}
\hline Medida Kaiser-Meyer-Olkin & 0.713 \\
Prueba de & Aprox. Chi-cuadrado & 215.19 \\
esfericidad de & Gl & 6 \\
Bartlett & Sig. & 0.000 \\
\hline
\end{tabular}

Fuente: Elaboración propia, a partir de datos arrojados en SPSS V. 24.

Source: Own elaboration, based on data provided in SPSS V. 24.

Con respecto al análisis factorial por medio de componentes principales realizado para la variable de comportamiento innovador, se tiene que los 4 ítems aplicados inicialmente a la muestra de estudio fueron agrupados en un solo componente. Así mismo, la prueba de alfa de Cronbach 
El comportamiento innovador en empresas: una visión desde la autonomía y la disponibilidad de tiempo de los empleados de una empresa del sector transporte en Tamaulipas

indica un grado "bueno" con relación a la confiabilidad de la escala de medida con un valor de 0.811 (George y Mallery, 2003).

Cuadro 4. Matriz de componentes para innovación.

Chart 4. Rotated component matrix for innovation variable.

\begin{tabular}{|c|c|c|}
\hline Variable & Ítems & Factor 1 \\
\hline \multirow{4}{*}{ Innovación } & $\begin{array}{l}\text { D1.1. Cantidad de nuevas líneas de productos o servicios desde } 2010 \text {, donde el valor } 1 \text { representa } \\
\text { ninguna nueva línea de productos o servicios; el nivel } 5 \text { representa muchas nuevas líneas. }\end{array}$ & 0.809 \\
\hline & $\begin{array}{l}\text { D1.2. Nivel de cambios en las líneas de productos o servicios, donde el valor } 1 \text { representa } \\
\text { cambios en menor naturaleza y el nivel } 5 \text { cambios dramáticos en las líneas de productos o } \\
\text { servicios. }\end{array}$ & 0.760 \\
\hline & $\begin{array}{l}\text { D1.3. Postura de los altos directivos ante las innovaciones, donde el valor } 1 \text { significa un fuerte } \\
\text { énfasis de los directivos en la comercialización de productos/servicios ya probados y el valor } 5 \text { un } \\
\text { fuerte énfasis en investigación y desarrollo, liderazgo t }\end{array}$ & 0.833 \\
\hline & $\begin{array}{l}\text { D1.4. Nuevas técnicas para hacer frente a la competencia, donde el valor } 1 \text { significa que muy rara } \\
\text { vez mi organización, es la primera en introducir nuevos productos/servicios, técnicas } \\
\text { administrativas, tecnologías operativas, etc.; y, el valor } 5 \text { significa }\end{array}$ & 0.801 \\
\hline \multirow{3}{*}{$\begin{array}{c}\text { Otros } \\
\text { indicadores }\end{array}$} & Auto-valores & 2.569 \\
\hline & Porcentaje de la varianza & 64.236 \\
\hline & Alpha de Cronbach & 0.811 \\
\hline
\end{tabular}

Fuente: Elaboración propia, a partir de datos arrojados en SPSS V. 24.

Source: Own elaboration, based on data provided in SPSS V. 24.

Una vez agrupados los componentes, verificada la validez de los constructos y la existencia de correlaciones previas entre las variables, se procedió a realizar un análisis de correlación para determinar en qué medida se relacionan las variables independientes con el fenómeno de innovación empresarial.

Con lo anterior se determinó que la variable "disponibilidad de tiempo" o "tiempo libre" no es significativa con relación al fenómeno abordado, lo anterior se fundamenta en los resultados de Alpkan et al. (2010) quien en investigaciones previas comprobó que, contrario a otros estudios en la materia, la disponibilidad de tiempo no influye en el pensamiento creativo o el desarrollo de innovaciones de los trabajadores. 
Cuadro 5. Análisis de correlación.

Chart 5. Correlation analysis.

\begin{tabular}{|ccccc|}
\hline & Autonomía & $\begin{array}{c}\text { Tiempo } \\
\text { Libre }\end{array}$ & Innovación \\
\hline Autonomía & $\begin{array}{c}\text { Correlación de } \\
\text { Pearson } \\
\text { Tiempo Libre }\end{array}$ & 1 & 0.000 & $.266^{* *}$ \\
& $\begin{array}{c}\text { Sig. (bilateral) } \\
\text { Correlación de } \\
\text { Pearson } \\
\text { Sig. (bilateral) }\end{array}$ & 0.000 & 1.000 & 0.001 \\
\hline Innovación & $\begin{array}{c}\text { Correlación de } \\
\text { Pearson } \\
\text { Sig. (bilateral) }\end{array}$ & $.266^{* *}$ & 0.127 & 0.127 \\
\hline & 0.001 & 0.133 & 1 \\
\hline
\end{tabular}

Fuente: Elaboración propia, a partir de datos arrojados en SPSS V. 24. Source: Own elaboration, based on data provided in SPSS V. 24.

Por su parte la variable independiente "autonomía" se presenta con una baja correlación positiva con respecto a la innovación. Por lo general las correlaciones bajas se deben a la existencia de otros elementos no considerados en el modelo o a que realmente no exista una relación de causalidad en los datos (Barnett y Seth, 2014). Se asume que al trabajar con percepciones se está sujeto a un error en la varianza común en los datos por lo que resulta pertinente verificar que las propiedades psicométricas de los mismos son las adecuadas y que su medición es precisa.

Una prueba que permite verificar si la matriz de los ítems se encuentra afectada por el sesgo de la varianza común es el Test de Harman (Podsakoff, MacKenzie, Lee y Podsakoff, 2003). En esta prueba fueron incluidos en un único análisis factorial la totalidad de los ítems del cuestionario utilizando el método de componentes principales, sin rotación y forzando a un único factor. De acuerdo con Podsakoff et al. (2003) existirá un problema de varianza común cuando el único factor extraído supere el $50 \%$ de varianza compartida, por lo que se puede apreciar en el cuadro siguiente que no fue el caso para los ítems de esta prueba. 
El comportamiento innovador en empresas: una visión desde la autonomía y la disponibilidad de tiempo de los empleados de una empresa del sector transporte en Tamaulipas

Cuadro 6. Test de Harman.

Chart 6. Harman's Test.

\begin{tabular}{|c|c|c|c|c|c|c|}
\hline \multirow{2}{*}{ Componente } & \multicolumn{3}{|c|}{ Autovalores iniciales } & \multicolumn{3}{|c|}{$\begin{array}{c}\text { Suma de las saturaciones al cuadrado de } \\
\text { la extracción }\end{array}$} \\
\hline & Total & $\begin{array}{c}\% \text { de la } \\
\text { varianza }\end{array}$ & $\begin{array}{c}\% \\
\text { acumulado }\end{array}$ & Total & $\begin{array}{c}\% \text { de la } \\
\text { varianza }\end{array}$ & $\begin{array}{c}\% \\
\text { acumulado }\end{array}$ \\
\hline 1 & 3.607 & 30.058 & 30.058 & 3.607 & 30.058 & 30.058 \\
\hline 2 & 2.091 & 17.429 & 47.487 & & & \\
\hline 3 & 1.816 & 15.133 & 62.62 & & & \\
\hline 4 & .894 & 7.448 & 70.069 & & & \\
\hline 5 & .699 & 5.826 & 75.894 & & & \\
\hline 6 & .647 & 5.390 & 81.284 & & & \\
\hline 7 & 5.86 & 4.884 & 86.168 & & & \\
\hline 8 & .486 & 4.048 & 90.216 & & & \\
\hline 9 & .427 & 3.556 & 93.772 & & & \\
\hline 10 & .324 & 2.703 & 96.216 & & & \\
\hline 11 & .234 & 1.948 & 98.423 & & & \\
\hline 12 & .189 & 1.577 & 100.000 & & & \\
\hline
\end{tabular}

Fuente: Elaboración propia, a partir de datos arrojados en SPSS V. 24. Método de extracción "componentes principales".

Source: Own elaboration, based on data released in SPSS V. 24. Extraction method "main components".

Una vez realizadas las pruebas anteriores se procedió a realizar el análisis de regresión lineal múltiple que permitió determinar el efecto de la autonomía y la disponibilidad de tiempo otorgados a los trabajadores en el nivel de innovación en la empresa.

El cuadro siguiente indica el resumen del modelo $Y_{i}=b_{0}+b_{1} X_{1}+b_{2} X_{2}+e_{i}$, donde $\mathrm{R}$ representa los valores de coeficiente de correlación entre las variables, $\mathrm{y}, \mathrm{R}^{2}$ es el coeficiente de determinación que indica la proporción de mejora causada por el modelo propuesto, es decir, la proporción de variabilidad de la variable dependiente $\left(Y_{i}\right)$. Con lo anterior, se tiene que el primer modelo explica sólo un 7\% de la innovación en la empresa y es significativo. El segundo modelo no resulta significativo por lo que se deduce nuevamente que la disponibilidad de tiempo no impacta en el comportamiento innovador en la empresa. 
Cuadro 7. Resumen del modelo.

Chart 7. Model Summary.

\begin{tabular}{|cccccccccc|}
\hline Modelo & $\mathbf{R}$ & $\begin{array}{c}\mathbf{R} \\
\text { cuadrado }\end{array}$ & $\begin{array}{c}\mathbf{R} \\
\text { cuadrado } \\
\text { ajustado }\end{array}$ & $\begin{array}{c}\text { Estándar de } \\
\text { la } \\
\text { estimación }\end{array}$ & $\begin{array}{c}\text { Cambio } \\
\text { en } \mathbf{R} \\
\text { cuadrado }\end{array}$ & $\begin{array}{c}\text { Cambio } \\
\text { en } \mathbf{F}\end{array}$ & gl1 & gl2 & $\begin{array}{c}\text { Sig. } \\
\text { Cambio en } \\
\mathbf{F}\end{array}$ \\
\hline 1 & $.266^{\mathrm{a}}$ & 0.071 & 0.064 & 0.97339494 & 0.071 & 10.640 & 1 & 140 & 0.001 \\
2 & $.294^{\mathrm{b}}$ & 0.087 & 0.074 & 0.96842579 & 0.016 & 2.440 & 1 & 139 & 0.121 \\
\hline
\end{tabular}

Fuente: Elaboración propia, a partir de datos arrojados en SPSS V. 24. A) Predictores: (Constante), Autonomía; B) Predictores: (Constante), Autonomía, Tiempo Libre; C) Variable dependiente: Innovación.

Source: Own elaboration, based on data provided in SPSS V. 24. A) Predictors: (Constant), Autonomy; B) Predictors: (Constant), Autonomy, Free Time; C) Dependent variable: Innovation.

Los resultados expuestos en el cuadro de ANOVA indican el ajuste del modelo, en donde se tiene que F se representa por el residuo entre varianza sistemática y no sistemática. En este sentido los datos arrojados señalan una predicción relativa con respecto al fenómeno de innovación dado que F se encuentra situado por encima de 1 . Así mismo, el nivel de significatividad $(\mathrm{p}<0.05)$ indica la habilidad del modelo para la predicción de la innovación en la empresa. No obstante, se observa que el modelo que contempla sólo la autonomía resulta más significativo que el que analiza el efecto de ambas variables independientes.

Cuadro 8. ANOVA.

Chart 8. ANOVA.

\begin{tabular}{|ccccccc|}
\hline Modelo & & $\begin{array}{c}\text { Suma de } \\
\text { cuadrados }\end{array}$ & Gl & $\begin{array}{c}\text { Media } \\
\text { cuadrática }\end{array}$ & F & Sig. \\
\hline 1 & Regresión & 10.082 & 1 & 10.082 & 10.640 & $.001^{\text {b }}$ \\
& Residuo & 132.650 & 140 & 0.947 & & \\
& Total & 142.731 & 141 & & & \\
\hline 2 & Regresión & 12.370 & 2 & 6.185 & 6.595 & $.002^{\text {c }}$ \\
& Residuo & 130.361 & 139 & 0.938 & & \\
& Total & 142.731 & 141 & & & \\
& & & & & & \\
& a partir & &
\end{tabular}

Fuente: Elaboración propia, a partir de datos arrojados en SPSS V. 24. A) Predictores: (Constante), Autonomía; B) Predictores: (Constante), Autonomía, Tiempo Libre; C) Variable dependiente: Innovación.

Source: Own elaboration, based on data provided in SPSS V. 24. A) Predictors: (Constant), Autonomy; B) Predictors: (Constant), Autonomy, Free Time; C) Dependent variable: Innovation.

Por otro lado, los valores Beta del modelo expuesto en el cuadro siguiente se sitúan para la autonomía en un 0.266. Lo anterior señala que el incremento del nivel de autonomía en la empresa por una unidad de desviación estándar incrementaría la innovación en un 0.266 de la 
desviación estándar de la constante. Es importante señalar que esta relación entre variables se presenta baja a pesar de la significancia de la prueba; así mismo, que en el caso de la variable de disponibilidad de tiempo, el efecto de relación no podría asumirse en el sentido de que su valor no se presenta significativo.

Cuadro 9. Coeficientes de regresión.

Chart 9. Regression coefficients.

\begin{tabular}{|ccccccc|}
\hline Modelo & & B & $\begin{array}{c}\text { Error } \\
\text { estándar }\end{array}$ & Beta & T & Sig. \\
\hline 1 & (Constante) & 0.005 & 0.082 & & 0.063 & 0.950 \\
& Autonomía & 0.267 & 0.082 & 0.266 & 3.262 & 0.001 \\
2 & (Constante) & 0.005 & 0.081 & & 0.063 & 0.950 \\
& Autonomía & 0.267 & 0.082 & 0.266 & 3.279 & 0.001 \\
& $\begin{array}{c}\text { Tiempo } \\
\text { Libre }\end{array}$ & 0.127 & 0.082 & 0.127 & 1.562 & 0.121 \\
& & & & & \\
\hline
\end{tabular}

Fuente: Elaboración propia, a partir de datos en SPSS V. 24. A) Predictores: (Constante), Autonomía; B) Predictores: (Constante), Autonomía, Tiempo Libre; C) Variable dependiente: Innovación. Source: Own elaboration, based on data provided in SPSS V. 24. A) Predictors: (Constant), Autonomy; B) Predictors: (Constant), Autonomy, Free Time; C) Dependent variable: Innovation.

\section{Conclusiones}

Se reconoce que la dinámica comercial actual sugiere que las empresas busquen formas alternativas de alcanzar un mejor desempeño financiero o de mercado y en esta búsqueda un método eficiente para lograr resultados positivos es el desarrollo de una postura orientada a la innovación. Es por ello que la delimitación de nuevas formas que promuevan tal comportamiento resulta altamente necesaria.

En este sentido en el presente estudio se planteó el supuesto de que algunos factores internos como la autonomía y la disponibilidad de tiempo brindado a los trabajadores como parte de un proceso de empoderamiento o descentralización, se relacionaban de manera positiva y significativa en la innovación en la empresa.

Lo anterior había sido demostrado en investigaciones previas (e.g. De Villiers-Scheepers, 2012; Kuratko, 2005, 2010, 2014; Moriano, Topa, Molero y Levy, 2009). Sin embargo, la literatura basada en evidencia empírica para el caso de economías emergentes como México carece de resultados para la gran mayoría de los sectores y regiones por lo que demostrar la relación de las variables planteadas en el estudio, en el contexto de empresas de servicios de 
transportación situadas en el noreste de México, contribuye a la construcción de una teoría que aún se encuentra en desarrollo.

Gracias a los resultados de la investigación se reconoce que la autonomía de los trabajadores se relaciona de forma positiva y significativa a la innovación en empresas del sector y territorio analizados, por lo que se respaldan las deducciones de las investigaciones realizadas en el contexto de las economías desarrolladas y, por ende, no se rechaza la primera hipótesis (H1) de esta investigación.

No obstante, los resultados de la regresión lineal aplicada a las observaciones de la muestra indican que la disponibilidad de tiempo en los empleados no se encuentra asociado al desarrollo de innovaciones por lo que la segunda hipótesis (H2) se rechaza. Se ha de aclarar que el tiempo libre otorgado a los empleados y su relación con el comportamiento innovador se encuentra en función del sector y el territorio al que pertenece la empresa, por lo que se puede conjeturar que la variable es irrelevante en sectores y territorios específicos o bien, que podría ser explicada por una variable interviniente como el caso de aquellas que competen a la motivación o a las características emprendedoras de los individuos.

Algunos ejemplos de lo anterior se presentan en las investigaciones de Alpkan et al. (2010), quienes analizaron la relación del tiempo libre y la innovación en empresas manufactureras de Turquía y descartaron la existencia de una asociación entre las mismas. En contraste a esos hallazgos Agrawal, Catalini, Goldfarb y Luo (2018) realizaron una investigación sobre el papel que juega el tiempo libre en la asignación endógena del tiempo y esfuerzo en proyectos innovadores en el sector educativo de Estados Unidos y descubrieron que la holgura de tiempo incide de forma positiva en el desarrollo de nuevas ideas y proyectos. Este último descubrimiento, se asemeja a lo expuesto por Gordart, Görg y Hanley (2016), quienes analizaron el grado de innovación en productos y procesos entre empresas comerciales que trabajan por contratos de horario libre y las que trabajan con contratos de tiempos establecidos y encontraron que las primeras tienden a tener entre un 12 y un 15\% más de probabilidades de mejorar sus productos y entre un 7 y $8 \%$ más de probabilidades de emprender procesos innovadores. Lo anterior sugiere que los autores encontraron una relación positiva entre la adopción de términos contractuales basados en la confianza y la innovación, sin embargo, esta relación es impulsada por el control y autogestión del tiempo, las cuales son características inherentes de los individuos y no de la empresa. 
El comportamiento innovador en empresas: una visión desde la autonomía y la disponibilidad de tiempo de los empleados de una empresa del sector transporte en Tamaulipas

En lo que respecta al sector y territorio específico de la presente investigación es importante aclarar que se analizó a la empresa líder del sector, por lo que se puede conjeturar que el grado de autonomía de los empleados sí se relaciona al comportamiento innovador, pero el tiempo libre brindado a los empleados de empresas del mismo rubro de la zona noreste de México, no se encuentra relacionado con el desarrollo de esa conducta en la empresa.

Es relevante señalar que este estudio presenta algunas limitaciones importantes relacionadas con la cantidad de elementos internos que pueden incidir en el desarrollo de una conducta innovadora. Entre estos aspectos se tienen aquellos de carácter normativo, culturales y de estilos gerenciales o de toma de decisiones, así como condiciones exógenas relacionadas a condiciones favorables o desfavorables que propicien la innovación en empresas del rubro. Se deduce que una comprensión más profunda del fenómeno estará sujeta al estudio de modelos más complejos.

\section{Agradecimientos}

Agradecemos al Consejo Nacional de Ciencia y Tecnología en México, al Centro de Enseñanza Técnica y Superior de Baja California y a la Universidad Autónoma de Tamaulipas.

\section{$\underline{\text { Referencias }}$}

Agrawal, A., Catalini, C., Goldfarb, A. y Luo, H. (2018). Slack Time and Innovation. Organization Science, 29 (6), 1056-1073. DOI: 10.1287/orsc.2018.1215

Alpkan, L., Bulut, C., Gunday, G., Ulusoy, G. y Kilic, K. (2010). Organizational support for intrapreneurship and its interaction with human capital to enhance innovative performance. Management Decision, 48 (5), 732-755. doi: 10.1108/00251741011043902

Antoncic, B. (2007). Intrapreneurship: A comparative structural equation modeling study. Industrial Management and Data Systems, 107 (3), 309-325. DOI: $10.1108 / 02635570710734244$

Antoncic, B. y Hisrich, R. D. (2001). Intrapreneurship: Construct refinement and cross-cultural validation. Journal of Business Venturing, 16 (5), 495-527. DOI: 10.1016/S08839026(99)00054-3

Anderson, J. y Markides, C. (2007). Strategic innovation at the base of the pyramid. MIT Sloan Management Review, 49, 83-8. 
Barnett, L. y Seth, A. K. (2014). The MVGC multivariate Granger causality toolbox: a new approach to Granger-causal inference. Journal of Neurocience Methods, 223, 50-68. DOI: 10.1016/j.jneumeth.2013.10018

Baruah, B. y Ward, A. (2014). Metamorphosis of intrapreneurship as an effective organizational strategy. International Entrepreneurship and Management Journal, 11 (4), 811-822. DOI: $10.1007 / \mathrm{s} 11365-014-0318-3$

Burgelman, R. A. (1984). Designs for corporate entrepreneurship. California Management Review, 26 (3) 154-166. DOI: 10.2307/41165086

De Villiers-Scheepers, M. J. (2012). Antecedents of strategic corporate entrepreneurship. European Business Review, 24 (5), 400-424. DOI: 10.1108/09555341211254508

Duncan, W. J., Ginter, P. M., Rucks, A. C., y Jacobs, T. D. (1988). Intrapreneuring and the reinvention of the corporation. Business Horizons, 31 (3), 16-21

Galván-Vela, E., y Sánchez-Limón, M. (2018). Autonomía y recompensas como factores organizacionales detonantes de la actividad intraemprendedora. Ciencias Administrativas Teoría y Praxis, 13 (2). Recuperado a partir de: https://cienciasadmvastyp.uat.edu.mx/index.php/ACACIA/article/view/109

Galván-Vela, E. y Sánchez-Limón, M.L. (2019). Factores organizacionales relacionados con el comportamiento intraemprendedor. Innovar: Revista de Ciencias Administrativas y Sociales, 29 (71), 55-68. DOI: 10.15446/innovar.v29n71.76395.

George, D. y Mallery, P. (2003). SPSS for Windows step by step: A simple guide and reference. 11.0 update $\left(4^{\mathrm{a}}\right.$ ed.). Boston, MA: Allyn \& Bacon.

Gordart, O. N., Görg, H. y Hanley, A. (2016). Trust-Based Work Time and Innovation: Evidence from Firm-Level Data. ILR Review, 70 (4), 894-918. DOI: 10.1177/0019793916676259

Guth, W. D. y Ginsberg, A. (1990). Guest Editors' Introduction: Corporate Entrepreneurship. Strategic Management Journal, 11, 5-15.

Hair, J. F., Anderson, R. E., Tatham, R. L. y Black, W. C. (1999). Análisis Multivariante. España: Pearson Prentice Hall.

Halme, M., Lindeman, S. y Linna, P. (2012). Innovation for Inclusive Business: Intrapreneurial Bricolage in Multinational Corporations. Journal of Management Studies, 49 (4), 743 784. DOI: $10.1111 / \mathrm{j} .1467-6486.2012 .01045 . \mathrm{x}$ 
El comportamiento innovador en empresas: una visión desde la autonomía y la disponibilidad de tiempo de los empleados de una empresa del sector transporte en Tamaulipas

Hornsby, J. S., Kuratko, D. F., Holt, D. T. y Wales. W. J. (2013). Assessing a Measurement of Organizational Preparedness for Corporate Entrepreneurship. Journal of Product Innovation Management, 30 (5), 937-955.

Hornsby, J. S., Kuratko, D. F., Shepherd, D. A. y Bott, J. P. (2009). Managers' corporate entrepreneurial actions: Examining perception and position. Journal of Business Venturing, 24 (3): 236-247

Hornsby, J. S., Kuratko, D. F. y Montagno, R. V. (1999). Perception of internal factors for corporate entrepreneurship: A comparison of Canadian and U.S. managers. Entrepreneurship Theory and Practice, 24 (2), 9-24

Hornsby, J. S., Kuratko, D. F. y Zahra, S. A. (2002). Middle managers' perception of the internal environment for corporate entrepreneurship: assessing a measurement scale. Journal of Business Venturing, 17 (3), 253-273. DOI: 10.1016/S08839026(00)00059-8

Hoskisson, R. E., Hitt, M. A., Wan, W. P. y Yiu, D. (1999). Theory and research in strategic management: Swings of a pendulum. Journal of Management, 23 (3), 417-456.

Ireland, R. D., Covin, J. G. y Kuratko D. F. (2009). Conceptualizing corporate entrepreneurship strategy. Entrepreneurship Theory and Practice, 33 (1), 19-46. DOI: 10.1111/j.15406520.2008.00279.x

Kearney, C., Hisrich, R. D. y Roche, F. W. (2010). Change management through entrepreneurship in public sector enterprises. Journal of Developmental Entrepreneurship, 15 (4), 415-437. DOI: 10.1142/S1084946710001646

Kearney, C., Hisrich, R. D. y Antoncic, B. (2013). The mediating role of corporate entrepreneurship for external environment effects on performance. Journal of Business Economics and Management, 14 (1), 328-357. DOI: 10.3846/16111699.2012.720592

Kerlinger, F. N. y Lee, H. B. (1983). Investigación del comportamiento. México: McGrawHill.

Knight, G.A. (1997). Cross-cultural reliability and validity of a scale to measure firm entrepreneurial orientation. Journal of Business Venturing, 12, 213-225.

Kuratko, D. F. (2010). Corporate Entrepreneurship: An Introduction and Research Review. In Z. J. Acs y D. B. Audretsch (Eds.). Handbook of Entrepreneurship Research, pp. 129-163. Springer New York. Recuperado de http://link.springer.com/chapter/10.1007/9781-44191191-9_6 
Kuratko, D. F. y Audretsch, D. B. (2013). Clarifying the domains of corporate entrepreneurship. International Entrepreneurship and Management Journal, 9 (3), 323-335. DOI: $10.1007 / \mathrm{s} 11365-013-0257-4$

Kuratko, D. F., Hornsby, J. S. y Covin, J. G. (2014). Diagnosing a firm's internal environment for corporate entrepreneurship. Business Horizons, 57 (1), 37-47. DOI: 10.1016/j.bushor.2013.08.009

Kuratko, D. F., Ireland, R. D., Covin, J. G. y Hornsby, J. S. (2005). A model of middle level managers' entrepreneural behavior. Entrepreneurship Theory and Practice, 29 (6), 699716.

Kuratko, D. F., Montagno, G. y Hornsby, J. S. (1990). Developing an intrapreneurial assessment instrument for effective corporate entrepreneurial environment. Strategic Management Journal, 2 (Summer), 49-58

Laclette, J. (2012). Diagnóstico en ciencia, tecnología e innovación para el estado de Tamaulipas. Foro Consultivo, Científico y Tecnológico. México.

Martin, B. R. (2016). Twenty challenges for innovation studies. Science and Public Policy, 43 (3), 432-450. DOI: 10.1093/scipol/scv077

Michelli, J. y Oliver, R. (2017). Empresas de software en México y sus vínculos de desarrollo local. Problemas del Desarrollo, 48 (190), 37-59. DOI: 10.22201/iiec.20078951e.2017.190.57534

Miller, D. (1983). The Correlates of Entrepreneurship in Three Types of Firms. Management Science, 29 (7), 770-791. DOI: 10.1287/mnsc.29.7.770

Montero, R. (2013): Test de Causalidad. Documentos de Trabajo en Economía Aplicada. Universidad de Granada. España.

Moriano, J. A., Topa, G., Molero, E. y Lévy, J. (2011). The influence of transformational leadership and organizational identification on intrapreneurship. International Entrepreneurship Management, 10 (2), 103-119. DOI: 10.1007/s11365-011-0196-X

Morse, C. W. (1986). The delusion of intrapreneurship. Long Range Planning, 19 (6), 92-95.

Nelson, R. (1991). Why do firms differ, and how does it matter? Strategic Management Journal, $12,61-74$.

Nelson, R. y Winter, S. G. (1982) An evolutionary theory of economic change. Cambridge: Harvard University Press. 
El comportamiento innovador en empresas: una visión desde la autonomía y la disponibilidad de tiempo de los empleados de una empresa del sector transporte en Tamaulipas

Nelson, R. y Winter, S. G. (2002). Evolutionary theorizing in economics. Journal of Economic Perspectives, 16 (2), 23-46

Organización para la Cooperación y el Desarrollo Económico (2005). Manual de Oslo. Guía para la recogida e interpretación de datos sobre innovación: La medida de las actividades científicas y tecnológicas ( $3^{\mathrm{a}}$ ed.). OCDE, EUROSTAT.

Parker, S. C. (2011). Intrapreneurship or entrepreneurship? Journal of Business Venturing, 26 (1), 19-34. DOI: 10.1016/j.jbusvent.2009.07.003

Pérez, C. (2009). Técnicas de Análisis de Datos con SPSS 15. España: Pearson Prentice Hall.

Podsakoff, P. M., MacKenzie, S. B., Lee, J. Y. y Podsakoff, N. P. (2003). Common method biases in behavioral research: a critical review of the literature and recommended remedies. Journal of Applied Psychology, 88 (5), 879-903. DOI: 10.1037/0021-9010.88.5.879

Schumpeter, J. (1934). The Theory of Economic Development. Cambridge, MA: Harvard University Press.

Schumpeter, J. (1942). Capitalism, socialism and democracy. Nueva York: Harper.

Teece, D. y Pisano, G. (1994). The dynamic capabilities of firms: an introduction. Industrial and Corporate Change, 3 (3), 537-556.

Teece, D. J., Pisano, G. y Shuen, A. (1997). Dynamic capabilities and strategic management. Strategic Management Journal, 509-533.

Turró, A., Urbano, D. y Peris-Ortiz, M. (2014). Culture and innovation: The moderating effect of cultural values on corporate entrepreneurship. Technological Forecasting and Social Change, 88, 360-369. DOI: 10.1016/j.techfore.2013.10.004

Wyk, R. y Adonisi, M. (2012). Antecedents of corporate entrepreneurship. South African Journal of Business Management, 43 (3), 65-78

Yoguel, G., Barletta, F. y Pereira, M. (2013). De Schumpeter a los neoschumpeterianos: viejas y nuevas dimensiones analíticas. Problemas del Desarrollo, 174 (44), 35-59.

Zahra, S. A. (1991). Predictors and financial outcomes of corporate entrepreneurship: An exploratory study. Journal of Business Venturing, 6 (4), 259-285. DOI: 10.1016/08839026(91)90019-A

Zahra, S. A. (1993). Environment, corporate entrepreneurship, and financial performance: a taxonomic approach. Journal of Business Venturing, 8 (4), 319-40. DOI: 10.1016/08839026(93)90003-N 